\title{
La marque du père
}

Sur une notation de Pu Songling à propos de sa naissance

The Mark of the Father. On a Notation by Pu Songling concerning his Birth 父親的烙印一一關于蒲松齡出生的注釋

\section{Rainier Lanselle}

\section{OpenEdition \\ Journals}

Édition électronique

URL : http://journals.openedition.org/extremeorient/211

DOI : 10.4000/extremeorient.211

ISSN : 2108-7105

\section{Éditeur}

Presses universitaires de Vincennes

\section{Édition imprimée}

Date de publication : 1 janvier 2012

Pagination : 83-112

ISSN : 0754-5010

\section{Référence électronique}

Rainier Lanselle, "La marque du père », Extrême-Orient Extrême-Occident [En ligne], Hors-série | 2012, mis en ligne le 01 janvier 2015, consulté le 01 mai 2019. URL : http://journals.openedition.org/ extremeorient/211 ; DOI : 10.4000/extremeorient.211 


\title{
La marque du père. Sur une notation de $\mathrm{Pu}$ Songling à propos de sa naissance
}

\author{
Rainier Lanselle
}

\begin{abstract}
«À l'heure même où l'arc fut suspendu pour annoncer ma naissance, mon défunt père fit un rêve: il vit un disciple de Gautama, décharné et d'aspect maladif, dont la robe laissait une épaule découverte, entrer dans la pièce. Un emplâtre de la taille d'une pièce de monnaie, rond, était collé près de son sein. À son réveil, j'étais né. Or il se trouvait que je présentais une marque encrée qui s'accordait exactement à ce rêve.»
\end{abstract}

$\mathrm{Pu}$ Songling, «Auto-mémoire de Liaozhai » (18 année Kangxi, 1679) ${ }^{1}$.

La littérature savante sur Les Notes de l'étrange de Liaozhai (Liaozhai Zhiyi), l'opus magnum de Pu Songling (1640-1715), s'étend à perte de vue. Elle a approché ce monument par tous les abords imaginables, des plus strictement philologiques aux plus interprétatifs, étudiant ses thèmes, sa langue, explorant son discours sur le lien social ou le rapport entre les sexes, émettant hypothèses à foison sur l'anthropologie dont relève son univers surnaturel, familial, ou documentant l'imaginaire du système des concours à partir de ce qu'il en dit. La galerie de ses personnages-types a fait l'objet d'analyses variées, souvent pertinentes : fantômes et apparitions, animaux, femmes et épouses, lettrés neurasthéniques, mandarins, religieux... la liste n'en finirait pas, des travaux chinois ou occidentaux, qui ont fait avancer notre compréhension de cette œuvre et de son auteur au cours des vingt dernières années, y multipliant les angles de vue ${ }^{2}$.

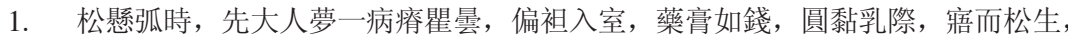
果符墨. Pu Songling et Zhang Youhe (éd.) (1978): vol. I, 2.

2. Pour ne citer que quelques titres : Barr (1984, 1985, 1986, 1989, 2007a, 2007b); Zeitlin (1993, 2007); Chiang (2005); Chang et Chang (1998); McMahon (2006, 2009) : 22-30; Huntington (2003); Weightman (2006); Yuan Shishuo et Xu Zhongwei (2000); Wang Zhizhong (1990); Revue Pu Songling yanjiu 蒲松龄研究 (Zibo 淄博, Shandong). La 
Pourtant je n'en connais pas qui le prenne par le thème précis du père, et ferait de celui-ci l'une des problématiques centrales du livre.

Écartons tout malentendu: la figure du père apparaît bien sûr dans de nombreux récits de $\mathrm{Pu}$ Songling, comme membre d'à peu près n'importe quelle constellation familiale au sein de laquelle se déroule une histoire. Pour autant il ne retient pas beaucoup l'attention des critiques, qui le voient comme un protagoniste nécessaire de telle ou telle problématique, sans en faire la visée même de l'enquête. Qu'il s'agisse de savoir comment le père malheureux est vengé ${ }^{3}$, comment il reçoit un signe avant-coureur avant la naissance d'un enfant ${ }^{4}$, ou encore qu'on entreprenne de décrire l'horreur qui aurait atteint l'«ordre patriarcal $^{5} \gg$, pour ne citer que quelques exemples de ce qui pourrait être une litanie, le père est présent mais le plus souvent sans être autrement défini. Il est, comme personnage, trop stéréotypé pour qu'il soit besoin d'en dire beaucoup plus que ce qu'il est: le père, justement. Rien de comparable avec le luxe qui caractérise l'évocation ou l'analyse des figures féminine ou maternelle, et qui correspond en vérité au prodigieux inventaire qui en est fait dans l'œuvre de $\mathrm{Pu}$ Songling. Femme fantôme, héroïne, apparition sous toutes les formes possibles ${ }^{6}$, vierge ou prostituée, maman ou fille facile, fatale dans tous les sens du terme, elle est toujours remarquable ${ }^{7}$, et il y a toujours quelque chose à en dire. Quand il est question de gender, c'est presque toujours du sien qu'il s'agit, quand bien même les sexes seraient, avec $\mathrm{Pu}$ Songling, en proie à la «dislocation» (Huntington) : puisque c'est le féminin, avec son incorporation multiforme, qui domine l'imaginaire, le masculin n'en apparaît que mieux comme en creux, en tant que contrecoup typiquement déclassé et falot; l'homme type des Notes de l'étrange est bien plus un fils qu'un père ${ }^{8}$. Symptomatiquement, l'étude de la famille et des valeurs éthiques dans les récits du Liaozhai revient à évoquer, comme protagonistes, des notions ou règles faisant loi, telles la piété filiale ou la bonne entente entre les frères, diverses autres vertus confucéennes stéréotypées, des conduites plus ou moins adéquates de fils, et... des détails soudain prolixes sur la conduite des femmes et les hiérarchies compliquées entre ces dernières, qu'elles soient belles-mères, brus ou épouses de divers rangs. Du père, rien à signaler, pourrait-on dire, et lorsque Chun-shu Chang et Shelley Chang font

traduction française d'André Lévy est la seule à ce jour complète du Liaozhai zhiyi dans une langue occidentale: Pu Songling et Lévy (2005).

3. Guan $\mathrm{Wu}(1996,1997)$.

4. Zeitlin (1993): 48 et n.18, 236.

5. Chiang (2005): 97-113.

6. Zeitlin (2007).

7. McMahon (2006), (2009): 22-30, (2007); Barr (1989).

8. Huntington (2000); McMahon (2009); Zeitlin (1993): 98-131. 
l'analyse de ces aspects, leur évocation, presque paradigmatique, du conte qui ouvre le recueil, ne sait peut-être pas à quel point elle est pertinente: c'est une histoire entre un fils, une mère et... des dieux ${ }^{9}$. De père, point.

Pourquoi cette difficulté à faire le portrait du père ? Est-ce qu'il manquerait une théorie du père, et qu'une théorie de la femme (soutenue par l'essor américain, dans les dernières décennies, des gender studies) serait plus aisée à manier? Le problème doit être plus structurel. De façon typique, plusieurs des contributions à la même livraison d'Extrême-Orient, Extrême-Occident où paraît la présente étude ont trouvé plus adéquat de faire ressortir la question paternelle par contrecoup, à partir des contours de problématiques adjacentes: Mark Lewis, par exemple, le fait depuis le rapport mère-fils en élaborant à partir d'une psychologie des «liens émotionnels» qu'il implique; Keith McMahon à partir des séductions et des violences du gynécée où n'apparaît que d'assez loin, à travers la perversion, l'ombre de ce qui serait une figure - plutôt inquiétante - du père ${ }^{10}$. Ces contournements ne sont pas fortuits. C'est que la femme est une figure beaucoup plus imaginaire que celle du père: elle fournit en bonne logique à l'imagination, comme à la peinture psychologique, car il n'en va pas $\mathrm{du}$ «Féminin» comme du «masculin». Le père, lorsqu'il est traité en tant que père, relève de l'ordre symbolique: il n'a pour ainsi dire pas de psychologie, et le champ dont il relève n'est guère celui de la description sur laquelle on puisse beaucoup s'attarder. Il faut donc le prendre par un autre bout pour voir comment il s'inscrit, et c'est là que nous nous tournons vers cette citation de Pu Songling que nous avons placée en exergue à cet article.

Celle-ci est tirée de l'«Auto-mémoire de Liaozhai» («Liaozhai zizhi»), que l'auteur rédigea en 1679. Un peu plus étendu, l'extrait de l' "Auto-mémoire» qui nous intéresse dit ceci:

À l'heure même où l'arc fut suspendu pour annoncer ma naissance, mon défunt père fit un rêve : il vit un disciple de Gautama, décharné et d'aspect maladif, dont la robe laissait une épaule découverte, entrer dans la pièce. Un emplâtre de la taille d'une pièce de monnaie, rond, était collé près de son sein. À son réveil, j'étais né. Or il se trouvait que je présentais une marque encrée qui s'accordait exactement à ce rêve. De plus, lorsque j'étais enfant, j'étais maigre et souvent malade, et parvenu à l'âge d'homme mon sort ne fut guère brillant. La désolation de ma demeure n'avait rien à envier à une résidence monastique, et les labours de l'encre et du pinceau étaient d'aussi petit profit que le bol à aumône. Plus d'une fois, me grattant la tête, je

9. Chang et Chang (1998) : 101-115. Il s'agit du conte Lz1 : «Kao Chenghuang» 考城隍, «L'examen au poste de dieu des Murs et des Fossés», un conte à la première personne. Sur le caractère de typicité de ce conte, parmi les plus précoces d'une collection dont la composition s'étend sur plus de trois décennies: Barr (1985).

10. Ci-dessous Pu songling \& Zhang Youhe (1978): vol. I, 243 sqq et 159 sqq. 


\section{Rainier Lanselle}

m'interrogeais : celui qui méditait face au mur était-il vraiment ma propre incarnation dans une existence antérieure? Sans doute fallait-il qu'il y eût quelque déficience dans les causes fondamentales, pour qu'incapable d'obtenir les fruits par lesquels j'aurais pu transcender ma condition, je me trouvasse ballotté au gré du vent, et finisse ainsi, fleur tombée dans la fosse d'aisance. Troubles ô combien, les Six chemins de l'existence! mais qui osera dire qu'ils n'ont point leur raison ${ }^{11}$ ?

Ce texte a été traduit avec des bonheurs divers, selon le degré de littéralité et d'attention à un énoncé où chaque mot, chaque caractère compte. Judith Zeitlin, qui consacre tout un chapitre à ce «Liaozhai zizhi », à cette «Self-Introduction» qu'elle nomme «a crazy quilt of disembodied images», en traduit plusieurs extraits $^{12}$. Le début du passage est rendu chez elle:

At the hour of my birth, my late father had a dream: a gaunt, sickly Buddhist monk, whose robe left one shoulder bare, entered the room. A plaster round as a coin was pasted on his chest. When my father awoke, I had been born, with an inky birthmark that corroborated his dream ${ }^{13}$.

André Lévy, dans sa traduction de ce qu'il rend par «Autochronique», et qui accompagne la traduction intégrale de l'œuvre, surtraduit avec hardiesse mais de façon astucieuse comme nous le verrons :

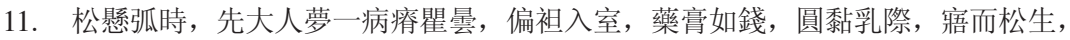
果符墨誌。且也, 少贏多病, 長命不猶。門庭之淒寂, 則冷淡如僧; 筆墨之耕 耘, 則蕭條似鉢。每搔頭自念, 勿亦果是吾前身耶？蓋有漏根因，未結人天之 果; 而隨風蕩墮, 竟成藩洞之花。茫茫六道, 何可謂無其理哉! Pu Songling et Zhang Youhe (éd.) (1978): vol. I, 2. Xuanhu 懸弧, « arc suspendu», est une expression métonymique désignant la naissance d'un garçon, à partir d'une allusion au Liji 禮記, «Neize» 內則 : «Quand un enfant naît, si c'est un garçon, un arc est suspendu à gauche de la porte d'entrée» (子生, 男子設弧於門左); voir Hanyu da cidian 漢語大詞典 (1997) : 4395. Bimo zhi gengyun 筆墨之耕耘, «les labours de l'encre et du pinceau» fait référence à l'expression proverbiale yifen gengyun, yifen shouhuo 一分耕耘, 一 分收穫, «pour tant de labour, autant de récolte». Pu Songling fait évidemment allusion aux maigres revenus que lui procurera son pinceau, lui qui ne parviendra jamais à passer avec succès les examens supérieurs. Voir Jiaoyubu guoyu tuixing weiyuanhui (1994), entrée 《耕耘》. Mianbiren 面壁人, «celui qui méditait face au mur», fait allusion à la manière dont Bodhidharma ( $\mathrm{v}^{\mathrm{e}}$ siècle) médita pendant neuf ans face à un mur, au monastère de Shaolin, au mont Song. L'expression désigne par extension tout moine. N.B. : Pu Songling se désigne ici par le seul monosyllabe de Song 松, puisque Ling 齡 est un beifen 輩分, un marqueur de génération partagé dans sa fratrie, ses trois derniers frères ayant pour nom Boling 柏齡, Songling 松齡 et Heling 鶴齡 - Yuan Shishuo et Xu Zhongwei (2000): 13.

12. Zeitlin (1993): 43-58; dans sa traduction, Zeitlin reprend partiellement H. A. Giles (infra, n. 15).

13. Ibid.: 48 . 
À ma naissance, mon père avait rêvé d'un disciple malade et décharné du Bouddha Gautama, qui entrait dans la chambre l'épaule dénudée, une rondelle de pommade grosse comme une sapèque collée sur le sein. À son réveil, j’étais né et je portais en effet la marque de l'encre noire de ces chroniques ${ }^{14}$.

D'autres traductions publiées sont toutes inférieures aux deux précédentes, ne seraient-ce que parce qu'elles rejettent le caractère mo 墨, «encre», qu'elles trouvent sans doute trop bizarre, au profit de son quasi-homographe hei 黑, de signification adjacente: «noir ${ }^{15} »$.

Or ce détail compte, comme j'ai pu le relever ailleurs ${ }^{16}$, de même qu'une autre particularité, concernant, elle, l'autre caractère présent dans l'expression mozhi 墨誌, «marque encrée». Ce caractère y est affecté d'une nuance graphique guère possible à rendre, pour le coup, en traduction. Au lieu de graphier zhi («marque») avec le caractère 痣, c'est-à-dire avec le marqueur sémantique des symptômes et des affections corporelles (广), et signifiant «grain de beauté» ou «marque de naissance», il l'écrit, profitant d'une licence de substitution graphique qui ne manque pas de précédents et repose naturellement sur une étymologie commune ${ }^{17}$, avec le $z$ hi comprenant la clé de la parole: 誌 et signifiant «marquer», « consigner», « note», « notation», « mémoire». C'est le même zhi

14. Pu Songling et Lévy (2005) : vol. I, 23-24. André Lévy donne sa perception de l'«Automémoire» in Lévy (1992).

15. John Minford dans sa traduction partielle du Liaozhai zhiyi: «My father,/When I was born,/And the bow hung at his door,/Dreamed of a sickly Buddha,/Cassock bare at the right shoulder,/Entering the room,/On one breast/A plaster round like a coin./He woke from sleep,/And saw on his own newborn child/A black patch. » (Pu Songling et Minford (2006b), écran 5998). Minford ajoute dans une note qui ne craint pas les raccourcis : «A black patch: In other words, the child was the reincarnation of the Buddha. 'Black patch' can also be take to mean Ink Records. The tales themselves are predestined.» (ibid., écran 6197). Allan Barr: "When the bow was hung at my father's door, he dreamed that a sickly-looking Buddhist priest, but half-covered by his stole, entered the chamber. On one of his breasts was a round piece of plaster like a cash; and my father, waking from sleep, found that I, just born, had a similar black patch on my body.» (Barr (1986): 106). Herbert A. Giles: «When the bow was hung at my father's door, he dreamed that a sickly-looking Buddhist priest, but-half covered by his stole, entered the chamber. On one of his breasts was a round piece of plaster like a cash and my father, waking from sleep, found that I, just born, had a similar black patch on my body.» (Pu Songling et Giles (trad.) (1916) : XV). Jacques Dars et Chan Hing-ho : «Pour moi, à ma naissance, mon défunt père rêva qu'un maladif et hâve disciple de Gautama entrait dans la pièce, la tunique dégageant l'épaule droite, un emplâtre de la taille d'une sapèque collée sur son sein; quand il s'éveilla, j'étais né... avec en effet une marque noire identique.» (Dars et Chan (2001): 200-201).

16. Pu Songling et Lanselle (2009): 40.

17. Hanyu da zidian (1993): 1654, § 5 sous l'entrée «誌». 
誌 que l'on retrouve dans Liaozhai zhiyi 聊齋誌異, le titre de l'ouvrage, comme d'ailleurs dans «Liaozhai zizhi» 聊齋自誌, «Auto-mémoire de Liaozhai» («Liaozhai », la «Retraite de l'oisiveté», étant le nom de la bibliothèque de $\mathrm{Pu}$ Songling, et partant, son surnom).

Cette particularité a été remarquée par Zeitlin, qui la voit comme signifiante:

Thus the proof-mark of his father's premonitory dream explains both the etiology of his vocation as recorder of the strange and the source of his privileged insight into such matters ${ }^{18}$.

La même particularité explique le saut sémantique qu'entend effectuer Lévy en condensant, dans sa traduction/interprétation, ce «texte» originel, de destinée, et le texte même de ce qui sera l'œuvre d'une vie, et qui, littéralement, nous fait comprendre Lévy, fera d'un symptôme langage.

Ces interprétations méritent qu'on y souscrive. Mais encore faudrait-il les étayer théoriquement, pour qu'elles ne restent pas que simples intuitions. Avant de faire cette tentative, il faut justifier pourquoi cette brève notation de $\mathrm{Pu}$ Songling sur sa naissance vaut qu' on en approfondisse l'analyse. Il y a à cela plusieurs raisons. D'abord parce que cette notation est très étrange. Je n'en connais pas qui, dans les formes par nature très codifiées, pour ne pas dire ritualisées, des paratextes liminaires, pousse aussi loin dans un sens personnel, révélant un détail de l'intimité du corps de l'auteur. Non que dans la tradition des préfaces, dans laquelle l' «Auto-mémoire »s'inscrit, les impressifs, les subjectivèmes, les considérations les plus intimes sur le destin de l'auteur ne soient légion. Elles se disent selon des codes que Pu Songling maîtrise mieux que personne et dont il fait montre ici : avec des considérations sur le karma, la réincarnation et les origines obscures d'un destin traversé, par exemple; mais aussi par des procédés stylistiques, qui sont ceux du hiératisme archaïsant, et enclin à une certaine dépersonnalisation du discours, propre à la prose parallèle (pianwen) dont il fait usage. Ce style donne à cet «Auto-mémoire», ou «auto-préface» («Zizhi») un aspect très ciselé que les commentateurs ne manquent jamais de rappeler ${ }^{19}$, et il est par nature moins intimiste que le style communément en usage du guwen, plus spontané. Dans ce contexte, cette notation, qui survient tout d'un coup chez Pu Songling sur un point de réel de son corps, prend un relief tout spécial, donnant à l' «Auto-mémoire» une coloration à part. Une autre raison pour accorder une attention particulière à ses propos est que 1' «Auto-mémoire» est un document unique, puisqu'il est le seul dans lequel Pu Songling parle en long de la composition des Notes de l'étrange, une entreprise qui par ailleurs

18. Zeitlin (1993): 48.

19. Zeitlin (1993): 43-58; Pu Songling et Lévy (2005): 11. 
domine largement l'ensemble de son œuvre d'écrivain puisque sa composition s'étale sur une période de quarante ans, peut-être plus ${ }^{20}$. C'est un texte bref, à l'économie stricte, dont on peut penser qu'il ne comporte rien de superflu pour son auteur. Enfin, le «Liaozhai zizhi», rédigé au terme d'une décennie sans doute intensément consacrée à la composition des Notes, n'a été suivi par aucune autre auto-préface de même type, alors même que, datant de 1779, elle précède de peut-être trente ans les dernières pièces du recueil. Autrement dit rien, pour autant qu'on sache, n'est jamais venu supplanter ou corriger l' «Auto-mémoire de Liaozhai » en termes de paratexte de Pu Songling lui-même concernant la composition de l'œuvre.

L'extrait que j'ai relevé sur cette circonstance particulière de la naissance de Pu Songling est donc unique. Il combine trois éléments : 1 . un rêve du père au moment de la naissance du sujet; 2 . un destin plutôt malheureux qui s'accorde avec cette prémonition portée par le discours du père; 3 . une marque physique qui vient, sur le corps même du sujet, attester ce discours. Cette marque en ellemême ne «dit» rien, mais elle n'est pas moins signifiante, puisque toute une vie subséquente vient la doter de signification. Si peu fervent bouddhiste que $\mathrm{Pu}$ Songling ait été en réalité, on peut assurément relever, à un niveau culturel comme à un niveau personnel, «the archetypal nature of the dream ascribed to $\mathrm{Pu}$ Songling's father» $\left(\right.$ Zeitlin $\left.^{21}\right)$.

Que ce passage soit porteur, pour Pu Songling qui nous le dit lui-même, d'une explication globale concernant l'ensemble de sa vie constitue une information précieuse. Cette explication est de plus indicatrice de la direction que prend son œuvre, le choix du surnaturel étant présenté comme subséquent un peu plus loin dans le «Zizhi ${ }^{22} »$. Cependant, pour fonctionner, le discours de destinée lié au rêve du père aurait pu se limiter au mécanisme classique fourni par l'explication «rationnelle» du rêve : le rêve du père concernant le fils à naître

20. Le Liaozhai zhiyi aurait été composé sur une période allant du début des années 1670 à 1711 , selon des déductions tirées des événements contemporains les plus précoces et les plus tardifs dont il est fait mention dans le corps de l'œuvre. Pu Songling et Lévy (2005) : vol. I, 9-18; Pu Songling et Lanselle (2009) : 26-30 ; Barr (1984-1985). Selon Ma Ruifang, la composition des contes étranges pourrait avoir commencé dès 1664, soit un an après la mort du père de Pu Songling, alors que celui-ci a seulement 24 ans : Ma Ruifang 马瑞芳 (1986): 33. Également Yuan Shishuo et Xu Zhongwei (2000): 176-234, 291-294.

21. Zeitlin (1993): 236-237, n. 18. Notations de Gao Heng 高㹸 (1612-1697), ami de Pu Songling, en 1790, sur le bouddhisme de ce dernier: $C f$. ibid., p. 49. Thème du parent rêvant de moines au moment d'une naissance: Wu (1990): 164, 174 (deux cas de rêves de mères), cité par Zeitlin (1993): 236-237, n. 18.

22. Il revendique l'héritage de la tradition des zhiguai, en citant le nom du Youming lu 幽 冥錄, le recueil attribué au prince Liu Yiqing 劉義慶 (403-444). 
révèle une causalité karmique, et un banal travail d'analogie du rêve à la réalité s'ensuit, qui vient confirmer point par point cette «révélation», donnant sens au destin et à ses avanies. Autrement dit, deux des trois éléments présents dans le passage relevé suffisaient. Pu Songling s'y montre d'ailleurs conforme à une convention narrative présente dans nombre de récits fictionnels de l'époque, y compris le Liaozhai $^{23}$. Alors pourquoi avoir relevé cette singularité de la marque sur le corps, si c'est pour la laisser échapper à tout commentaire, comme si elle relevait d'un réel indicible? Tout se passe comme si, du rêve du père, cette marque constituait l' «ombilic», ce reste présent dans chaque rêve, selon Freud, et qui en constitue la partie irréductible à toute interprétation.

Mon hypothèse est que cette présence signifiante du père, dont la marque est comme un garant pour Pu Songling, constitue un élément essentiel de sa propre motivation d'écriture. C'est aussi un élément essentiel de la personne de Pu Songling, bien entendu. Mais la psychologie de Pu Songling n'est pas ce qui m'intéresse; c'est plutôt de tenter de repérer certains éléments qui, dans l'œuvre, se comprennent mieux si l'on tient compte de la présence structurelle de la référence paternelle. Pour cela, ce n'est pas tellement du père «réel» de $\mathrm{Pu}$ Songling que nous avons besoin ${ }^{24}$, mais de tout autre chose, qui a pour nom le père, et qui est beaucoup plus une fonction qu'une personne en particulier.

C'est là que prend sens le recours à ce monument théorique qu'est la problématique du père dans la psychanalyse lacanienne. Cette problématique constitue l'une des lignes de force du travail de Lacan d'un bout à l'autre de son enseignement. Reliée à une foule d'autres aspects conceptuels qui plus ou moins en dépendent (la question des origines, le refoulement, le langage, la loi, la sexuation, le nouage du Réel, du Symbolique et de l'Imaginaire...), il s'agit d'une ligne directrice particulièrement foisonnante, l'une des plus difficiles aussi. Le repérage de cette question du père par Lacan comme centrale pour la théorie analytique repose sur plusieurs textes fondateurs de Freud où s'articulent des questions autour de la loi, de l'interdit œdipien, de la castration, de la différence sexuelle (l'analyse du petit Hans, Totem et Tabou, L'Homme Mö̈se et le monothéisme par exemple ${ }^{25}$ ). Au vrai, «la question du père n'a jamais cessé de hanter l'œuvre freudienne ${ }^{26} »$. Il faudra cependant le travail d'intégration de la question de la langue comme située au cœur de la structure inconsciente pour que Lacan puisse réaliser une percée théorique et clinique qui dotera la fonction paternelle

23. P. ex.: Lz232 («Liu Liangcai» 劉亮采), Lz240 («Egui» 餓鬼), Lz339 («Yu Qu’e» 于去惡).

24. Sur le père réel de Pu Songling: Yuan Shishuo et Xu Zhongwei (2000): 12-15.

25. Freud (2003); Freud (1993); Freud (1986a).

26. Askofaré et Sauret (2004): 258. 
de qualités fondamentales pour la vie psychique. C'est que le nom du père ne sera rien de moins que la clé de voûte de l'organisation symbolique, ceci pour le sujet d'abord, puis par rapport à la loi - c'est-à-dire à toute loi d'organisation familiale ou sociale, en se rappelant que ce nom joue de façon indépendante du positionnement réel du père (biologique) dans une société donnée, voire même pour le sujet individuel.

Qu'est-ce qu'un père ? C'est un nom. Là se situe une différence fondamentale avec la mère, qui elle n'a aucun besoin de nomination puisque sa qualité de mère est obvie. En revanche, cette mère est la seule à pouvoir désigner à l'enfant son père, ce qui s'accomplit par une transformation qualitative qui change tout: du père biologique, on passe à l'inscription dans un ordre d'une tout autre nature, celui, symbolique, du langage. C'est là que les choses se compliquent énormément pour le sujet. Celui-ci n' «acquiert» ce père qu'au prix d'un bond concomitant, littéralement, dans l'inconnu de l'Autre (puisque le langage est son domaine), et rencontre, parmi quantité de problèmes posés par ce bond, celui de la garantie: qui me dit que l'Autre dit vrai (puisque dans le même temps cet Autre me signifie qu'il est marqué du manque, conséquence logique de ce qu'au-delà de lui il y a une loi, qui lui est extérieure, et avec laquelle il ne se confond pas)? Avec cette parole sur le père, qui exige sans contrepartie qu'on lui fasse crédit, est entraîné en fait tout l'ordre du langage, dont les bases supposent structurellement ce même risque posé au sujet, d'un Autre dont la garantie est incertaine, car trouée d'un manque - son inscription découle logiquement du dévoilement par la mère de son propre manque aux yeux de l'enfant, consubstantiel à la nomination du père («je ne suis pas tout : il y a un père »). Lacan comparera l'ordre symbolique du langage à un vaste tissu dont le signifiant fondamental, le «point de capiton», sera ce «Nom-du-Père » grâce auquel l'ensemble des signifiants tient en un réseau à peu près cohérent. À l'inverse, la psychose sera justement ce cas où, le nom du père, forclos, fait défaut au moment où il est convoqué, et présente un trou, dans lequel le sujet tombe littéralement: les signifiants privés de ce point de capiton, le réseau devient comme un tissu qui se défait, et c'est le délire, où le langage divague et les mots persécutent. Dans un autre approfondissement, c'est toujours avec pour visée une réponse à apporter aux problèmes posés par le Nom-du-Père que Lacan développera ses ultimes élaborations sur le nouage «borroméen» du Réel, du Symbolique et de 1'Imaginaire, et sur la manière dont le symptôme opère dans des structures, telle celle de Joyce, où ce nouage pourrait se défaire ${ }^{27}$. «L'interrogation Qu'est-ce que le père? est posée au centre de l'expérience

27. Lacan (1975), (2005); Aubert (1987); Brivic (2008); Askofaré et Sauret (2004): 274. 
analytique comme éternellement non résolue», selon Lacan ${ }^{28}$ : elle n'intéresse pas moins tout ce qui, sans être au premier chef analytique, est concerné par la psychanalyse «en extension», comme dans l'exemple qui a suscité ici mon intérêt, et fait signe vers une hantise en lien avec cette marque du père sur le corps ${ }^{29}$. J'ajouterai que ces problèmes relèvent de la structure psychique, du langage, et sont autant de facteurs situés en amont des formations culturelles: la critique courante, qui voit d'un mauvais œil l'approche des «choses chinoises » avec l'outil analytique, sous-entend toujours un exotisme a priori de ces dernières, position contre laquelle j'ai à peine besoin de préciser que je m'inscris en faux.

Comme on l'imagine, la littérature analytique sur la question du père est considérable $^{30}$, mais les quelques éléments théoriques ci-dessus rappelés nous suffiront pour l'heure pour une première approche de l'œuvre de Pu Songling à partir de cette énigme qu'il nous pose, dans sa notation sur sa naissance. Encore une fois, ce n'est pas «son» père biographique qui intéresse ici. C'est même à peine de lui, Pu Songling, qu'il s'agira pour le moment, même si, du besoin d'inscrire pour la postérité la mémoire de cette inscription signifiante sur son corps à la possible valeur de symptôme de ses «obsessions ${ }^{31}$ », bien des choses de sa structure subjective pourraient être évoquées. Elles n'ont pas besoin d'être développées pour l'heure. C'est le père entrevu sous quelques aspects de sa fonction qui retiendra ici notre attention. Car mon hypothèse est qu'il n'est pas possible que ce point nodal de son existence même, cette marque du père qu'il juge utile de révéler dans son unique préface à son œuvre, ne vienne pas, ailleurs dans l'œuvre même, faire sentir sa présence. Après tout, c'est lui-même qui nous dit qu'il y a un lien entre cette marque et son œuvre.

En vérité, c'est peu dire que Pu Songling nous donne l'embarras du choix : pour prendre les seules Notes de l'étrange, les ressources qu'il nous offre à cet égard sont d'un luxe presque infini. C'est dans ce recueil que je choisirai les quelques exemples qui suivent, dont les thématiques sont en lien avec la réussite sociale (y compris le thème des concours mandarinaux) et la filiation, deux domaines où se trouve toujours sous-jacente la question du père et de l'inscription de son nom.

28. Lacan (1994): 372 .

29. Sur le lien entre besoin d'inscription et psychose: Essaim, $n^{\circ}$ 16: Des folies et des æeuvres (2006/1).

30. Par exemple Porge (1997) fait référence; bonne synthèse dans Dor (2002) : vol. I, 89173, chap. II, «La métaphore paternelle comme carrefour structural de la subjectivité»; plusieurs questions centrales bien résumées: Zafiropoulos (2003), 179-216, «Le nom du père, la psychose et la phobie».

31. Notions de $p i$ 癖, de $c h i$ 癡; Zeitlin (1993): 61-97, III, «Obsession»; Weightman (2006). 
Comme je l'ai relevé plus haut, les analyses des contes du Liaozhai zhiyi ont souvent été motivées par les remarquables portraits de femme qu'on y trouve. C'est le cas par exemple de Lz415 («Shuchi», «Le fou des livres $\left.{ }^{32} »\right)$. Dans ce conte «La» femme, sa supériorité évidente sur le jeune homme qui la rencontre et auquel elle apprend tout des choses du sexe, ont fait l'objet de notations pertinentes, mais qui toutes passent à peu près entièrement sous silence la question du père ${ }^{33}$. Pourtant ce récit ne peut vraiment se comprendre qu'à la condition d'en tenir compte - et surtout pour ce qui est d'apprendre les choses du sexe ! Le conte est d'autant plus intéressant que le père est ici «présent» dans une circonstance qui intègre clairement la question du langage et des signifiants. Dans ce récit, le père est mort. Le jeune homme héros de l'histoire, Lang Yuzhu 郎玉柱, vit au milieu de la bibliothèque paternelle dans une attitude de totale dévotion vis-à-vis de celle-ci. La fortune familiale a beau s'être effondrée, pour rien au monde il n'en vendrait un seul volume. Le jeune homme voue une vénération particulière à une calligraphie de la main de son père de l' «Exhortation à l'étude» («Quan xue pian»), le fameux poème de l'empereur Zhenzong des Song (r. 997-1022) dont plusieurs vers sont devenus quasi proverbiaux, en particulier ceux qui disent: «Dans les livres tu trouveras maisons d'or fin» et «Dans un livre tu trouveras la fille dont le visage est semblable au jade» («Shuzhong zi you huangjin wu [...] Shuzhong zi you yan ru yu»). Le jeune homme est persuadé que ce qui est annoncé dans ces formules se réalisera réellement. C'est ainsi que lorsqu'il découvre par hasard au sommet d'une étagère un modèle réduit de chariot en métal doré, il se persuade que c'est là un début de réalisation des «maisons d'or fin» dont parle le poème. Malgré son énorme savoir livresque, Yuzhu échoue cependant régulièrement aux examens. À plus de trente ans, il n'a jamais approché une femme, et ne se met pas en recherche d'un parti, persuadé qu'il est que c'est bien «dans un livre» qu'il trouvera «la fille dont le visage est semblable au jade».

32. Pu Songling et Zhang Youhe (éd.) (1978): 1453-1458. Ce conte fait partie du fragment de la collection Bodmer de Genève, et à ce titre j'en ai donné une traduction : Pu Songling et Lanselle (2009) : 79-89. Voir égal. trad. Lévy, «Bibliomane»: Pu Songling et Lévy (2005): vol. II, 1596-1602, N.B.: dans le présent article, la numérotation des contes du Liaozhai zhiyi suit celle fixée par Barr (1984: 556-562); c'est l'ordre suivi dans l'édition cardinale du Liaozhai (Pu Songling et Zhang Youhe (éd.) (1978)), ainsi que dans la traduction française de Lévy (Pu Songling et Lévy (2005)). Dans la suite, je me dispenserai donc de renvois à ces deux dernières références.

33. McMahon (2009): 26-27, «The Man Who Learns Sex from the Woman»; Zeitlin (1993): 95-97; Weightman (2006) : 203; Weightman (2008) : 132-140, «The Book Fool» (donne une traduction paraphrasée du conte). 
Or, c'est précisement ce qui arrive. Au cours d'une lecture, il découvre un marque-page ayant la forme d'une beauté de soie découpe, qui soudain prend vie, petite femme haute d'un pied - puis apparemment capable d'atteindre taille humaine - merveilleusement belle qui lui déclare répondre au patronyme de Yan (nom de famille qui, par ailleurs, comme nom commun, signifie entre autres «visage», ou «teint») et au nom personnel de Ruyu («Semblable au jade»). Autrement dit, l'apparition de Yan Ruyu réalise littéralement la formule du poème: «Dans un livre tu trouveras yan ru yu», i.e. «[la fille dont le] visage est semblable au jade». L'apparition entreprend alors de faire l'éducation sexuelle du jeune homme, qui dans ce domaine ignorait tout. Elle a beaucoup de mal à lui faire délaisser ses livres, s'efforçant de le délurer par la musique, le jeu et autres agréments sociaux, lui expliquant même que s'il rate les examens, c'est justement parce qu'il est trop livresque. Ils vivent ainsi deux ans, elle lui fait découvrir les plaisirs de la chambre, lui donne même un fils, mais finit par lui annoncer que leur bonheur risque fort de se terminer prématurément, à moins qu'il n'accepte de disperser sa bibliothèque. Il refuse: «Cette bibliothèque qui est votre pays natal? Qui est ma vie même? Mais vous n’y pensez pas! » lui répond-il. À quelque temps de là, la nouvelle que le jeune homme vit en ménage avec un être surnaturel finit par s'ébruiter. Le mandarin local, corrompu, en entend parler, et désireux d'approcher celle qu'on lui a décrite comme d'une beauté à couper le souffle, fait arrêter le jeune homme, le soumet à la torture et l'inculpe de sorcellerie. Apprenant que la fille se cache quelque part dans la bibliothèque, il fait incendier celle-ci: elle partira entièrement en fumée. Yan Ruyu ne réapparaîtra jamais. Le jeune homme parviendra par la suite à se faire disculper, réussira enfin les examens supérieurs et se fera nommer en poste dans la région du fonctionnaire corrompu, dont il instruira le procès et saisira tous les biens, y compris une concubine favorite qu'il fera sienne.

Qu'est-ce que le père? C'est un nom, avons-nous rappelé plus haut. Or, un nom n'est pas la chose même. Du père réel il n'y a (presque) rien à dire : c'est un père mort. Le père, qui assure une fonction dans le domaine symbolique, joue selon une logique de nom, lequel est bien plutôt «le meurtre de la chose ${ }^{34}$ » et est paré de caractéristiques d'équivocité partagées par le rôle que joue, dans le langage, la métaphore. La notion de métaphore est centrale dans la problématique lacanienne du Nom-du-Père, dont l'une des propositions fondamentales est que ce nom est un signifiant qui s'est, pour l'enfant, substitué à un autre signifiant. «C'est en tant que le père se substitue à la mère comme signifiant,

34. Lacan (1999b) : 317; Vanier (2000): 26-27; Dor (2002): vol. I, 117. 
que va se produire le résultat ordinaire de la métaphore ${ }^{35} »$ : si c'est avant tout en tant que «métaphore paternelle ${ }^{36}$ » que l'on est en droit de parler du père, c'est parce que, pour le sujet, le père est produit concomitamment au refoulement d'un autre signifiant, celui du désir de la mère. Le mécanisme est central dans la théorie analytique et porteur de conséquences en cascade. L'enfant menacé d'être pris dans la demande de la mère comme ne faisant qu'un avec cette demande, sans aucune perspective possible de subjectivation, découvre qu'il y a quelque chose que la mère désire et qui est au-delà de lui. Il découvre du même coup qu'il n'est pas tout ce qui comble la mère : il n'est pas le «phallus » maternel. C'est le père qui vient jouer ce rôle à la place de l'Autre, comme ce qui répond à l'énigme du manque de la mère - et que la mère pourrait bien désirer plus qu'elle ne le désire lui, l'enfant. Ce phallus n'est évidemment pas confondable avec le pénis du père : c'est au contraire, en quelque sorte, le pénis plus l'idée du manque. Comme tel, le phallus n'est rien d'autre que le signifiant du manque, quelque chose que le sujet ne peut donner, ne peut être (quand il s'accroche à cette idée, il tombe sous le coup de la structure perverse), et qui est à jamais hors de sa portée. Ce qui est important pour le récit qui nous occupe ici, c'est de comprendre que le père, comme le phallus, est un signifiant, et que sa métaphore apparaît dans un processus qui n'est pas séparable de la castration même. Le langage, qui est le fait d'un «sujet divisé», est par essence castré, étant structurellement une expérience de perte, comme l'est la découverte de la différence sexuelle, et ceci pour les deux sexes - puisque cette découverte se fait sur un mode cognitif qui ne saurait échapper à la fatalité d'être vécu comme perception du manque : là où il y a quelque chose chez l'un, cette même chose manquerait chez l'autre. L'enfant n'a cure de savoir qu'il ne manque rien à la femme pour être femme: il est comme le héros du conte du «Fou des livres», qui est essentiellement en enfant puisque à trente ans, il ne connaît rien de la différence sexuelle. Transposition dans le domaine du langage et de l'écriture - domaine que Pu Songling rattache, dans ce conte, de façon voyante à ce problème - : il se persuade que le patrimoine livresque et la calligraphie légués par le père sont la solution à tout manque. Partant, il leur refuse catégoriquement tout caractère d'équivocité (ils n'ont qu'un sens possible), en d'autres termes de castration (ils ne sont habités d'aucune altérité, i.e. il n'y a pas d'Autre), préservant en même temps le héros de l'histoire, par la vénération même qu'il porte à ce père en quelque sorte maintenu en vie artificielle à travers les écrits, de tout soupçon d'hostilité à l'égard de celui-ci. Les mots sont alors vidés de

35. Lacan (1998), 15 jan. 1958; Lacan ([1966] 1999) : 557, « D’une question préliminaire à tout traitement possible de la psychose».

36. Dor (2002): 118-120. 
toute qualité métaphorique, telle celle présente dans les images de l' «Exhortation à l'étude», et, selon un mécanisme classique chez Pu Songling, ils se laissent envahir par la chose même, ni plus ni moins que sous le mode de la hantise (même si, ici, Yan Ruyu est, par chance pour le héros, un bon fantôme). C'est là «[...] one of Pu Songling's most common mechanisms for generating fantasy, the literal realization of metaphoric language. Through this device, Pu Songling restores the shock of immediacy to a dead metaphor, reinvesting language itself with a new strangeness ${ }^{37} \gg$.

Il a été remarqué à bon droit que ce Fou des livres «[ressemblait] comme un frère chinois au Norbert Hanold de Gradiva» (Porret), c'est-à-dire que cette littéralité est lourde de la menace du délire ${ }^{38}$. La petite femme qui grossit et rapetisse à volonté, disparaissant et apparaissant depuis son «pays natal» de la bibliothèque, est l'une de ces femmes phalliques qui peuplent une part substantielle de l'œuvre de Pu Songling et mettent typiquement le héros en demeure de répondre à une demande impossible où l'alternative fatale est leur disparition. «[...] The nature of her attachment to the man is tenuous. [...] she is brazen and willfully cool; she comes and goes as she wishes » (avec «she»= la femme remarquable du Liaozhai zhiyi: «the female immortal, the fox, the ghost, the woman warrior or female knight-errant, or the shrew $\left.{ }^{39} »\right)$. Cette femme qui va et vient à sa convenance, mue par un caprice énigmatique, ne serait vraiment remarquable que pour qui n'aurait pas lu les observations de Freud sur le «fort$d a »$, ce jeu d'apparition/disparition par lequel l'enfant découvre qu'il y a autre chose que lui-même, que la mère désire ${ }^{40}$.

Si l'on se place de ce point de vue, il n'y a besoin d'élaborer aucune psychologie propre à l'homme Pu Songling : ce vers quoi pointent ces récits tient à la structure psychique, en général, de l'enfant par rapport à la question du désir de la mère. Ce que Pu Songling nous présente explicitement comme concernant «La» femme serait en fait plutôt à chercher, de façon inversée, du côté subjectif de l'enfant placé dans la situation de cette énigme. Je ferais l'hypothèse que ce n'est pas tant de la femme qui menace de s'en aller selon son caprice qu'il s'agit, que d'une place impossible à prendre pour le sujet lui-même. Le problème n'est pas tant: elle s'en va, que: je ne peux pas venir à la place où je crois qu'elle me demande. Et si cette place est impossible pour le sujet, c'est pour cette simple raison... qu'elle n'a pas de nom. La logique à l'œuvre ici est essentiellement une logique signifiante. «[...] Presque par définition, [...] l'enfant vient à la

37. Zeitlin (1993): 145, réflexion à partir de Lz305, «Meng lang» 夢狼, «Rêve de loups ».

38. Porret (2010): 252; Freud (1986b).

39. McMahon (2009): 23.

40. Freud (1981); Dor (2002): vol. I, 114-115. 
place d'un phallus qui manque à la mère : ce phallus, premier symbole, correspond au premier trauma, celui de la castration maternelle. Mais, par définition, il représente ce qu'il n'y a pas, et il reste donc innommable. Ainsi identifié à cette place, puisque c'est à ce titre qu'il a été désiré, un tel sujet ne porte pas de nom », écrit Gérard Pommier, qui ajoute: «L'enfant ne connaît d'abord que l'angoisse de la demande maternelle, c'est-à-dire celle de sa castration. Le père est supposé être l'agent de cette castration ${ }^{41}$.»

C'est la difficulté à trouver une substitution signifiante à cette question du désir de la mère qui pose comme central, chez $\mathrm{Pu}$ Songling, le problème du père. De ce père, il nous révèle dans sa préface à quel point il importe pour lui de l'inscrire quelque part (et pas n'importe où, mais sur ce corps dont nous aurons à dire un mot au moment de conclure). Autrement dit, le problème de cette femme qui va et vient à son gré, identifiée par maints auteurs comme typique du Liaozhai zhiyi, s'apparente, cette fois, à s'y méprendre, à un problème œdipien, c'est-à-dire à un problème de la castration de la mère par le père. «C'est eux ou moi », dit en substance Yan Ruyu à notre Fou des livres, qui n'est autre qu'un fou de littéralité: si tu veux me garder comme femme (elle est désormais la mère de son enfant), tu dois disperser les livres de la bibliothèque de ton père. En d'autres termes: tu dois faire advenir le père en acceptant de «le tuer» (ce que représenterait la disparition de cette bibliothèque qui en quelque sorte fait loi) pour que, comme sujet, tu puisses prendre son nom et trouver une place signifiante en regard du signifiant phallique de la mère. C'est ce processus, symbolique, qui a pour nom castration. Si l'agent (le père) en est réel, cet agent n'est pas la loi (i.e. ici : la bibliothèque et la calligraphie léguées par le père n'ont pas besoin d'être prises comme signification littérale), mais il la représente, comme un signifiant représente le sujet pour un autre signifiant, c'est-à-dire, cette fois, selon les lois, équivoques, du langage.

Le paradis délétère de Lang Yuzhu n'était qu'une tentative désespérée de satisfaire une femme phallique avec quelque chose capable de représenter le père, mais maladroitement pris dans un sens littéral. Heureusement pour lui, ce paradis prend fin grâce à quelqu'un qui lui apporte une aide inestimable, à savoir le mandarin corrompu, lorsque celui-ci vient trancher dans le vif de cette relation univoque, délirante, au langage dont Yan Ruyu était la formation symptomatique. Ce qui part en fumée, avec l'incendie provoqué de la bibliothèque paternelle, et avec Yan Ruyu elle-même, on peut dire que ce sont les théories sexuelles infantiles du héros, au sens que Freud donne à cette expression dans les Trois essais $^{42}$. La figure du père y advient d'ailleurs au milieu d'un débordement de

41. Pommier (2003) : 113-114.

42. Freud (2006). 
haine, désinhibée par les grands torts du mandarin à l'égard du jeune homme, qu'il a persécuté en son petit paradis. Car c'est bien dans une brèche œdipienne, que suppose une telle haine ${ }^{43}$, que le héros s'engouffre alors, pour aller in fine confisquer tous les biens de son ancien persécuteur et partir bras dessus bras dessous avec la concubine favorite de ce dernier ${ }^{44}$.

Plus encore, la nomination est inhérente au processus de castration qui accompagne ce travail de désidentification au phallus maternel - lequel était la marque de son état antérieur, où il ignorait la différence sexuelle. Pourquoi nomination? Pour parvenir à ses fins, il aura d'abord fallu que le héros trouve de quoi venir à bout de l'inexplicable succession d'échecs aux examens qui le poursuivait jusqu'alors, et qu'il réussisse à accéder au titre de jinshi («docteur»), condition pour pouvoir accomplir sa vengeance. Selon la formule bien connue, cette réussite correspond à la faculté pour un individu d'inscrire son nom propre sur le tableau doré des lauréats (jin bang ti ming), ce qui comporte son inévitable part de violence vis-à-vis du père ${ }^{45}$, que cette inscription vient supplanter - ce qui est in fine le sens que donne au freudien «meurtre du père » la logique lacanienne du père réduit au signifiant ${ }^{46}$.

Ce motif de la réussite ou de l'échec aux examens mandarinaux, et de l'iniquité du système, est comme on le sait l'un des thèmes majeurs du Liaozhai $z_{\text {zhiy }}{ }^{47}$, et sûrement l'un de ceux où la biographie de Pu Songling lui-même est le plus directement engagée: après tout, la seule chose qui se soit étendue sur une aussi longue durée dans sa vie que l'écriture du Liaozhai, mise à part sa situation de précepteur de la famille $\mathrm{Bi}$, est la succession, pendant pas moins de quarantesix ans, de ses échecs aux concours provinciaux ${ }^{48}$ ! Allan Barr, s'interrogeant après beaucoup d'autres sur les raisons pour lesquelles Pu Songling a toujours

43. Sur le mécanisme du «père aimé [qui] recouvre un père solidement haï d'une haine méconnue, inconsciente»: Bulat-Manenti (2009): 183.

44. Pour Frances Weightman, «His [celle de Lang Yuzhu] initial inappropriate childish mentality is replaced by a fully socialized adult view », mais sans qu'on sache très bien par quelle logique ce processus serait sous-tendu (Weightman (2008): 139). Mon appréciation du conte diffère également de la sienne, conte qu'elle juge «one of the most optimistic » du recueil (ibid.): le processus de perte dont le Fou des livres est l'emblème, pour être celui de tout un chacun, n'en est pas moins fondamentalement angoissant.

45. Pommier (2005): 23-24, 28.

46. Askofaré et Sauret (2004) : 266.

47. Elman (2000): 325-326, 359-365; Wang Fuchen (2010).

48. Éléments biographiques de Pu Songling en relation avec la candidature aux concours: Chang et Chang (1998) : 30-33, 125-134; Pu Songling et Lanselle (2009) : 25-26. L'échec au keju 科舉 serait à relier dès l'origine au choix que fait Pu Songling de l'écriture de l'étrange: Ma Ruifang 马瑞芳 (1986): 33. Pu Songling employé chez la famille de Bi Jiyou 畢際有 (1623-1693): Yuan Shishuo et Xu Zhongwei (2000): 99-125. 
échoué au xiangshi, à l'examen provincial, a sans doute raison de rappeler que les chances de réussite à son époque étaient de toute façon minimes et que l'irréussite de $\mathrm{Pu}$ n'avait à ce titre rien d'étonnant ${ }^{49}$ : cette vérité statistique ne liquide pas le problème des apparents actes manqués et atteintes somatiques intempestives qui font échouer Pu Songling, candidat au niveau d'ailleurs très élevé $^{50}$. Il n'est pas très risqué de faire l'hypothèse que les innombrables contes du recueil, parfois cruels ${ }^{51}$, où $\mathrm{Pu}$ Songling exprime sa haine du système du keju (des concours mandarinaux) recueillent la dérivation d'une part d'hostilité à l'endroit d'un père impossible à dépasser, ce père de Songling qui, issu d'une famille lettrée dont un membre eut le titre de jinshi («docteur»), abandonna la voie des concours pour celle du commerce après avoir échoué à l'examen le plus bas, celui de shengyuan ( $«$ bachelier $»)^{52}$.

L'inverse des contes formés sur ce thème de l'échec dans le système des concours et de l'hostilité à ce dernier serait à mon sens - c'est-à-dire selon une logique assez classiquement névrotique - à chercher non pas tant du côté des contes de réussite aux mêmes concours que du côté des contes, également remarquablement nombreux dans le Liaozhai, où apparaît une soumission «tendre» à la figure du père. On y trouvera régulièrement des motifs tels que ceux du sauvetage du père, voire de l'engendrement du père par le fils, selon un principe que résument Pierre Bruno et al.: «Quand l'enfant comprend qu'il doit sa vie à ses parents, il désire devenir un grand homme pour pouvoir payer sa dette. Il forme alors ce fantasme de sauver ses parents d'un danger menaçant leur vie. Quand ce fantasme s'applique au père, c'est le sens du défi qui y prédomine. Reste quelques cas où l'inclinaison tendre pour le père l'emporte sur le défi, et alors s'exprime le désir d'avoir le père comme fils ou encore d'avoir un fils

49. «The present study attempts to provide an answer to a question that is central to our understanding of Pu Songling's examination life: why did he fail ?» (Barr (1986) : 87).

50. En 1687, il tourne deux pages au lieu d'une, laissant une double page blanche (yиefu 越 幅) en recopiant son essai sur son cahier d'examen, ce qui est éliminatoire (Ma Ruifang 马瑞芳 (1986) : 218); l'empereur Qianlong tentera d'atténuer la sévérité de ces règles éliminatoires concernant les erreurs formelles (Pu Songling de «yuefu» (anon.)). En 1690, l'examinateur principal classe Pu Songling premier à l'issue de la première des trois épreuves, ce qui signifie qu'il se dispose à le recevoir major de la promotion (jieyuan 解元) à l'issue du concours. Mais Pu Songling tombe malade au milieu de la deuxième épreuve et est obligé d'abandonner (Ma Ruifang (2001): 219; Pu Songling et Lévy (2005): vol. I, 18-19).

51. P. ex. Lz26 («Sansheng» 三生, «Les trois vies $)$.

52. Alias xiucai 秀才. Données biographiques sur Pu Pan 蒲槃, père de Pu Songling: Ma Ruifang (1986) : 10 ; le grand-oncle Pu Shengwen 蒲生汶 a été jinshi en 1592 et souspréfet: Yuan Shishuo et Xu Zhongwei (2000): 11. 
comme son père ${ }^{53}$.» La binarité y joue souvent un rôle: «[...] contrairement au rêve, le mythe, comme le fantasme, est de nature paranoïde: il décompose là où le rêve condense, et la notion de double y est ainsi très présente ${ }^{54}$ ». À ce titre, on peut citer le remarquable Lz398 («Xi Fangping»), avec un fils qui, tel un moderne Mulian (Maudgalyāyana), n’hésite pas à mourir pour aller demander justice aux Enfers pour son père, supportant avec stoïcisme les plus cruels supplices, comme d'être grillé sur un lit de fer ou scié entre deux planches. Au retour, après le jugement favorable de Erlang shen, c'est le fils, premier revenu à la vie, qui ouvre le cercueil du père et redonne ainsi vie à ce dernier. La mort du père implique de faire vivre le père. Cette paternité inversée est un fantasme révélateur d'une réalité de structure : le fils fabrique du père symbolique à partir du père mort. L'imaginaire du père mort et de la dette à payer pour se rédimer par rapport à cette réalité se retrouve dans d'autres secteurs de la littérature narrative de la période pré-moderne, avec la figure prééminente de Nezha dans le Fengshen yanyi ${ }^{55}$. Bien des contes du Liaozhai traduisent un tel rapport d'ambivalence au père ${ }^{56}$, où se répondent thème de l'agressivité et soumission «tendre» à l'égard de celui-ci, voire identification féminine du sujet masculin avec désir d'engendrer un fils pour le père, et/ou... de réussir aux concours. Au moins deux contes qui brodent autour de ces thèmes (Lz339, «Yu Qu'e»; Lz240, «Egui », « Preta») associent ces derniers avec un autre, qui intéresse les propos de l' «Auto-mémoire de Liaozhai» dont nous sommes partis : le thème du rêve du père avant la naissance du fils - avec de surcroît, dans le second conte, ceux de la nomination et d'une marque à l'encre imposée sur le corps à l'insu du sujet ${ }^{57}$.

Comme le petit Hans de Freud, le héros du conte cité plus haut du «Fou des livres» est peut-être «fou», « hébété» (notion de $c h i^{58}$ ), mais il n'est pas si bête: dès avant l'effondrement de son petit paradis avec la fée des livres, sa croyance dans ce dernier ressortissait plus pour lui, apparemment, au fantasme qu'à l'hallucination, car il n'était pas dès le début sans un certain doute à l'égard de sa propre interprétation, par trop littérale, de la vérité des classiques, inter-

53. Bruno, Aouillé et al. (2008) : 106-107.

54. Ibid.

55. Ho (1988).

56. Rapport bien résumé par Pommier (2005).

57. En l'occurrence, la teinture d'une barbe. Sur ces contes et le thème de la «dreamvisitation that a father receives at the hour of his son's birth» : voir Zeitlin (1993): 48 et n. 18, 236-237. Thème de la soumission au père: voir par exemple Lz80 («Hongyu » 紅玉).

58. Voir le chap. que Frances Weightman consacre à ce conte: Weightman (2008): 132-140, «The Book Fool». 
prétation dont j'ai relevé qu'elle concernait une crainte de la castration. Selon Zeitlin, «[...] as soon as [Lang Yuzhu] realizes that the words of the ancients are lies, he swiftly passes the jinshi exams ${ }^{59}[\ldots] »$, et l'histoire se termine sur sa vengeance. Or, ce ne sont tout à fait ni cette temporalité ni ce dualisme (vrai/ pas vrai), avec un supposé «mensonge des Anciens» (Zeitlin) qui viendrait se révéler à lui. S'il y a dualisme, c'est au sens de l'obsessionalité, c'est-à-dire non exempt d'un tiers, et d'une division subjective. Il y a un épisode, tôt dans le récit - la chose est montrée de façon subtile -, où l'on voit bien comment il n'a maintenu sa croyance que par une volonté de la maintenir, et en la sachant fissurée - c'est la refente du sujet, où ce dernier s'aliène dans son propre discours ${ }^{60}$. Dans cette scène, la réponse au signifiant phallique (la sacralisation de la bibliothèque et de ses mots), c'est-à-dire au signifiant du manque, prend aussitôt la forme d'une convocation d'un Autre, et d'un Autre paternel. Mais cette convocation se fait sous une forme hostile (figuration dans le conte : à travers une amusante filouterie ${ }^{61}$ ), comme si cet Autre était mis au défi de rendre compte de ce manque. Or, si nous regardons l'ensemble du recueil, largement dominé par la figure, comme l'écrit McMahon, du «blank male $e^{62} »$, de l'homme falot, face à une femme souvent formidable, le reproche, c'est de ne pas être à la mesure du phallus de la mère, et, pour le père, de n'avoir pas su se mesurer au féminin.

Or, il est des plus classique pour la psychanalyse de concevoir qu'un homme puisse ériger une femme en place du signifiant, comme représentant phallique, c'est-à-dire la mette à la place de ce qu'il pense qu'elle n'a pas. Une formalisation comme le «Schéma $\mathrm{R}$ » de $\mathrm{Lacan}^{63}$ aide à conceptualiser et à comprendre la

59. Zeitlin (1993): 97.

60. Dor (2002): vol. I, 137.

61. «Un autre jour, comme il était monté avec une échelle à l'assaut d'une haute étagère il trouva, dans les volumes entassés sans ordre, un chariot doré d'un pied de long. Cette découverte lui causa une grande joie, car il y voyait de quoi vérifier le vers où il était question des “maisons d'or". Il montra sa trouvaille, qui révéla qu'en fait d'or fin elle était plaquée. Ceci n'alla pas sans lui causer un secret ressentiment à l'endroit des Anciens, qui semblaient s'être complu à le mystifier. À quelque temps de là, un superviseur de circuit, ancien camarade de promotion de son père, vint inspecter son district. Cet homme était pieux bouddhiste. Certains conseillèrent au secrétaire de lui offrir son chariot pour le placer dans une niche aux côtés de la statue du Bouddha. Le superviseur fut ravi du cadeau, et lui offrit trois cents onces d'argent et deux chevaux. N'était-ce pas là la preuve, pour le secrétaire comblé d'aise, que les "maisons d'or" et autres "équipages" du poème se voyaient vérifiés?» (Pu Songling et Lanselle (2009): 80). Les trois cents onces d'argent que le héros réussit à soutirer sont une somme considérable.

62. McMahon (2009): 27 ; passim.

63. «D'une question préliminaire à tout traitement possible de la psychose», in Lacan (1999a): vol. II, 31 ; Dor (2002): vol. II, 281-293. 
logique de ce mécanisme, qui implique la question de l'inscription phallique, dans un lieu qui relève du domaine de l'Autre. À partir de la renonciation au phallus de la mère, le désir du phallus peut fort bien porter, pour l'homme, vers une autre femme, parée des promesses du phallus, ceci dans une demande d'amour qui, par structure, est impossible à satisfaire, dans la logique du constat freudien selon lequel il y a dans la pulsion amoureuse quelque chose de foncièrement «non favorable à la réalisation de la pleine satisfaction ${ }^{64}$ ». « La» femme - la femme typique du Liaozhai - est alors inapprochable, ou, ce qui revient au même, aussitôt atteinte que perdue, pour la bonne et simple raison que c'est la nature même du signifiant du manque, qu'il lui revient d'incarner, que d'être pour toujours hors d'atteinte. Sous cet éclairage analytique, certains contes de Pu Songling deviennent ainsi particulièrement intelligibles. Par exemple Lz363 («Jinü», «La fileuse»), où figure cette scène prodigieuse de la jeune fille de beauté hors pair se révélant, telle une apparition divine, nimbée de lumière, derrière un store au jeune homme qui littéralement en tombe prosterné. Ce thème de l'écran, ce voilement (loin d'être unique dans le Liaozhai zhiyi ${ }^{65}$ ) relèvent tout entiers de la logique du fantasme, ce fantasme que rend Lacan par la formule $\$ \triangleright$ a, où l'objet cause du désir (a) n'est pas superposable à un objet qui serait spécularisable pour le sujet $(\$)^{66}$. Dans ce conte, l'accès à cet objet survient de façon fugace, comme si le voile était aboli : illusion aussitôt dissipée que formée, tant ce voilement est intrinsèque au fantasme ${ }^{67}$ («Elle lui apparut entièrement, comme si aucun n'écran ne l'en séparait plus $\left.{ }^{68} »\right)$. Un tel écran est indissociablement attaché au phallus, signifiant du manque par essence irreprésentable. Ce n'est pas pour autant, bien au contraire, que le sujet renoncera à y ériger en sa place... la femme elle-même, qui sera celle qui le sera (le phallus), à défaut de ce qu'un homme réel puisse l'avoir. Cette «autre femme», voilée, comme «puissance voyante» (Benslama ${ }^{69}$ ), a investi dans les accessoires de la beauté en vertu de cette logique par laquelle elle vient elle-même en place de ce signifiant phallique, selon une «mascarade»

64. Freud (2002): 64. Dans cet article, Freud fait la distinction entre le sentiment tendre et l'objet de l'attraction sexuel, qui peuvent différer. $C f$. la distinction chinoise entre qing 情, «sentiment», et yin 淫, «sensualité», sur laquelle voir McMahon (2009): 45.

65. Voir les deux contes liés: Lz469, «Wang Gui'an» 王桂菴/ Lz470 («Jisheng» 寄生). Dans ce dernier: voile couvrant le visage de la mariée donnant lieu à une illusion par rapport à l'objet d'élection; thème du regard.

66. Dor (2002): 81-89. «\$ $\$ \mathrm{a}$ » se lit « $\mathrm{S}$ barré poinçon de a», le «poinçon» étant une convention graphique destinée à montrer à la fois l'interrelation et la séparation. Quant au sujet, «\$», il est un sujet (S) affecté d'un barrage, celui de la division subjective.

67. Pluth (2008): 83, 87.

68. 無不畢現, 似無策幌之隔者. Pu Songling et Zhang Youhe (éd.) (1978): vol. III, 1223.

69. Benslama (2004): 191 et suiv. 
dont le sens est «symbolique ${ }^{70} »$. La jeune fille de Lz363 ( «Jinü») révèle un coin de cette vérité lorsqu'elle relève, littéralement, un coin du store pour laisser le jeune homme apercevoir... ses petits pieds chaussés de souliers brodés, venant contenter le désir du jeune homme qui «regrettait secrètement de n'avoir pu voir le bas de son corps $^{71} \gg$.

La scène comporte un aspect fétichiste, et la beauté, de par sa plénitude, est un déni du manque, et de ce qui le sous-tend, la castration. Aussi l'horreur de cette dernière menace-t-elle toujours de surgissement derrière chaque manifestation où la femme apparaît, puisqu'en bonne logique signifiante, la femme phallique «castre» l'homme. Les exemples en sont multiples dans le Liaozhai, parfois hallucinés. Dans Lz44 («Dong-sheng », «Le bachelier Dong»), le bachelier trouve dans son lit une fille d'une beauté divine, dont il entreprend d'explorer les parties génitales, pour y trouver, à sa plus grande terreur... une grosse queue velue! (c'était une renarde ${ }^{72}$ ). Dans Lz40 («Hua pi», « La peau peinte»), l'horreur du monstre hideux n'est que l'autre face cachée sous la peau de la beauté. Mais l'horreur relève-t-elle vraiment de l'autre face, ou n'est-ce pas la même, selon la logique möbienne que Lacan a considérée comme à l'œuvre dans toute opération signifiante? (Le «schéma $\mathrm{R}$ » évoqué plus haut en relève lui aussi.) Dans Lz252 («Jiangcheng»), on trouve ce dialogue, qui est une vraie perle tant il dévoile la logique d'horreur présente dans la beauté même:

[Gao Fan a une femme très belle mais terriblement tyrannique et dominatrice.]

Un jour qu'il buvait chez Ge, et qu'ils étaient quelque peu éméchés, Ge lui dit en le raillant:

- Mais qu'est-ce qu'elle a donc pour te faire si peur?

- Il y a en ce monde bien des choses inexplicables, répondit le jeune homme en riant. Ce qui me fait peur, eh bien c'est sa beauté ${ }^{73}$. J'en connais même qui ont des femmes dont la beauté est loin d'atteindre à celle de la mienne, et qui pourtant les craignent plus encore: n'est-ce pas encore plus déconcertant?

Ge fut gêné, et ne trouva quoi répondre ${ }^{74}$.

Cette tout-puissance de «La» femme est un thème qui revient sans cesse dans l'œuvre de Pu Songling. La hantise en est souvent représentée littéralement, le

70. Lacan (1973): 217; dans ce passage, il fait référence à l'apport de Joan Riviere sur la mise en scène de la féminité : Riviere (1929).

71。窲恨未睹下體. Pu Songling et Zhang Youhe (éd.) (1978): vol. III, 1223.

72. «[...]神仙不殊; 狂喜, 戲探下體, 則毛尾修然, 大懼 [...]». Pu Songling et Zhang Youhe (éd.) (1978): vol. I, 134.

73. 我之畏, 畏其美也.

74. Nos italiques. Pu Songling et Zhang Youhe (éd.) (1978): vol. II, 858. Je sais gré à K. McMahon d'avoir attiré notre attention sur ce passage important de ce dialogue dans McMahon (2009): 28. 
monstrueux de la femme surnaturelle du recueil obéissant à une logique d'autosuffisance: elle serait celle qui ne connaîtrait nul manque, n'obéirait à d'autre loi que celle de son propre caprice. Elle a des affinités avec cette Chose, ce das Ding freudien, objet perdu qui doit rester inaccessible et vers lequel le sujet serait englouti, dans une logique de jouissance ${ }^{75}$. Jouissance et destruction y vont typiquement d'un même pas. Comme réalité muette, elle est un point d'hallucination $^{76}$. Or, il n'y a à ce problème d'autre réponse que celle qui passe par le père, ce père dont le signifiant atteste qu'il y a du manque, comme le rappelle $\mathrm{Pu}$ Songling en associant, quand il parle de la marque du rêve paternel qu'il porte sur son sein, ce signifiant avec un dénuement monacal. La meilleure définition que l'on pourrait donner de cette marque serait ainsi de l'appeler la marque $d u$ manque. Cette association de la marque et du manque a été tôt identifiée par Lacan dans l'économie subjective du désir comme ce qui permet «l'accession à un certain étage du désir», «la marque [étant] le signe de ce qui supporte [une] relation castratrice». La marque est également un effet du signifiant, qui donne naissance au sujet, le sujet étant représenté par un signifiant (exemple: le don du nom), et la naissance de la subjectivité étant inséparable d'une part de manque puisqu'elle s'inscrit dans l'ordre du langage ${ }^{77}$. La marque du père est chez Pu Songling, dans son «Auto-mémoire», directement comprise comme la trace d'un personnage représentant sa subjectivité, personnage par essence castré dans son désir (le monachisme bouddhiste), et représentant pour le sujet à la fois son passé et son avenir (incarnation karmique, destin).

75. La Chose est l'objet du séminaire de Lacan L'Éthique de la psychanalyse: Lacan (1986).

76. Safouan (2001): vol. II, 146-147.

77. «J'insiste sur ce caractère de marque. D'ailleurs, en dehors de l'analyse, dans toutes ses autres manifestations interprétatives ou significatives, et bien certainement dans tout ce qui l'incarne cérémoniellement, rituellement, sociologiquement, la marque est le signe de ce qui supporte cette relation castratrice dont l'analyse nous a permis d'apercevoir l'émergence anthropologique. N'oublions pas les incarnations religieuses où nous reconnaissons le complexe de castration, la circoncision, par exemple, pour l'appeler par son nom, ou encore, dans les rites de puberté, telle forme d'inscription, de marque, de tatouage, en liaison avec une certaine phase qui se présente de façon non ambiguë comme l'accession à un certain étage du désir. Tout cela se présente toujours comme marque et impression.» (Lacan (1998) : 308, XVII, «Les formules du désir»). «Bien loin que le système de la demande soit parfait, à plein rendement ou à plein emploi, il s'introduit dans son arrière-plan l'effet du signifiant sur le sujet, la marque du sujet par le signifiant, et la dimension du manque introduite dans le sujet par ce signifiant. Ce manque introduit est symbolisé comme tel dans le système du signifiant comme étant l'effet du signifiant sur le sujet, à savoir le signifié.» (Ibid., p. 464, XXVI, «Les circuits du désir».) 
Les questionnements attachés à ces aspects sont complexes et leurs prolongements dépassent largement le cadre de cette enquête ${ }^{78}$, mais puisque cette dernière n'a d'autre objet que de montrer qu'il y a prégnance d'un problème paternel dans l'œuvre de Pu Songling, je terminerai en évoquant brièvement un dernier conte posant une question où le nom du père est essentiel : celle de la différence entre engendrement et filiation.

Dans Lz488 («Fang Wenshu»), le lettré Deng Chengde, sans enfant, séjourne à Yanzhou, loin de sa femme, comme précepteur. Il se met en ménage avec une femme encore une fois extraordinairement belle, Fang Wenshu, qui un jour s'est présentée spontanément chez lui. Ils vivent ensemble six ou sept ans, et elle lui donne un fils, qu'il nomme Yansheng, «Né à Yanzhou». Comme Deng lui propose de rentrer avec elle au pays, Fang Wenshu ne veut pas de la perspective d'être épouse secondaire; de plus elle n'a aucune envie d'avoir à supporter un bébé braillard. Puis elle lui dit froidement qu'elle s'en va, et disparaît avec l'enfant. (En partant, elle traverse la porte sans l'ouvrir, à quoi Deng comprend qu'elle était un être surnaturel.) De son côté, la femme de Deng, dame Lou, restée au pays, est seule depuis si longtemps qu'elle se croit veuve. C'est alors qu'elle reçoit la visite de Fang Wenshu, qui lui donne son propre enfant, dont elle (Fang) ne lui dit pas l'origine. Fang laisse également à Lou un sachet de drogue capable de provoquer la lactation. Puis elle disparaît. Lou ne tarde pas à aimer tendrement cet enfant, «comme s'il était sorti d'elle». Sans argent, elle tombe dans la misère, et reçoit un jour une nouvelle visite de Fang Wenshu. Elle craint que celle-ci ne soit venue lui réclamer son enfant, mais Fang la rassure, lui dit qu'elle est venue confirmer que l'enfant est bien à elle (Lou), et lui laisse de l'argent pour qu'elle puisse l'élever. Trois ans plus tard, le mari revient, enrichi par le commerce (il a abandonné sa condition de lettré), et découvre que sa femme a un fils. Elle lui dit comment elle l'a obtenu, qu'une inconnue le lui a laissé. C'est quand elle lui dit que «sa mère l'appelait Yansheng» que Deng Chengde comprend que c'est son propre fils. Il cherchera à retrouver Fang Wenshu, sans succès ${ }^{79}$.

Cette histoire est intéressante à plus d'un titre et pourrait se lire de plusieurs façons différentes $-\mathrm{y}$ compris en sortant du pacte narratif du merveilleux pour la regarder comme un habile maquillage de l'enfantement adultère de Lou en l'absence de son mari (après tout, elle se retrouve avec un enfant, et avec du lait pour l'allaiter, ce qui serait un peu beaucoup pour prétendre qu'une inconnue le lui a laissé...). Mais le père se laissera convaincre sans la moindre récrimination

78. Voir, par exemple, Assoun (2009): 337-349, II. XV, «Corps tracé et inconscient de la trace».

79. Sur ce conte: Barr (1985): 179-182. 
pour une seule question de nomination, celle où il est convoqué à l'endroit du père inconnu. Car il a beau savoir ce qui s'est réellement passé, ceci n'y fait rien: ce n'est pas par ce réel qu'il devient le père; seul un nom est capable de lui donner cette qualité. Il peut bien être le père réel de l'enfant, il est mis, grâce à l'habileté narrative de l'auteur, dans cette curieuse situation de ne pouvoir être ce père (une fois rentré dans son foyer), et surtout de ne pouvoir même se considérer comme tel (père et fils n'ont pas les moyens de se reconnaître après toutes ces années), tant qu'il n'a pas été nommé à cette place. Après tout, l'histoire aurait pu fonctionner tout aussi bien, non moins intéressante, avec Fang ne révélant pas à Lou le nom de l'enfant. L'histoire se serait terminée avec le couple Deng-Lou nanti d'un enfant de père décidément inconnu (même si c'était bien celui de Deng!), et cette fin aurait été différente, et beaucoup plus énigmatique pour tout le monde, y compris le lecteur. C'est du point de vue de l'enfant qu'il faut regarder les choses, et que le conte prend tout son intérêt. Pour cet enfant, ce qui s'en va comme mère, mû par un désir inconnu, lui revient comme père, comme nom. Cette mère unique, à la beauté sans pareil, indépendante, pour qui Deng ne représente aucune loi, en un mot phallique, est vouée à disparaître à jamais au profit d'une mère qui est son contraire, en ce qu'elle est, elle, soumise à la loi (traduction dans le langage du conte : à son mari, sur les frasques duquel elle n'a rien à dire). Précisons que le fait que cette épouse de Deng ait nommé le père inconsciemment (en nommant le fils à l'endroit du mari, elle nomme du même coup le père sans le savoir) est cohérent, si l'on veut bien lire l'histoire dans sa valeur métaphorique, avec le fait qu'elle est soumise, non pas à la personne réelle du bourgeois Deng, mais à ce qu'il vient ici représenter, et qui le dépasse: la loi du signifiant. Ce n'est qu'à partir de cette marque du père que l'enfant a apporté avec lui dans sa translation, et qui n'est autre que son nom (à lui, l'enfant), signifiant qu'il transporte sans même avoir à le prononcer de sa bouche, que le père est affecté à sa place, nommé comme père au moment où il réapparaît un beau jour. Dans ce conte, au-delà de leurs rôles biologiques naturels, le père et la mère qui ont engendré l'enfant jouent comme des places fonctionnelles, creusées par le symbolique. Ces place sont présentes, mais en attente d'être nommées, et quand elles le sont, les perspectives changent complètement. Le processus est celui d'une privation symbolique (réalisée par une nomination) d'un objet imaginaire (ici: la mère de beauté insurpassable, mais vouée à disparaître) par un agent réel (le père) : c'est ce processus même qu'on appelle castration dans la logique lacanienne du père réduit au signifiant.

Je dirai en conclusion que c'est ce dernier processus qui est en jeu dans la marque du père de $\mathrm{Pu}$ Songling. Cet indice que $\mathrm{Pu}$ donne dans sa préface est cohérent avec tant de «femmes phalliques» qui dominent son œuvre, et dont la figure imaginaire donne lieu à la galerie de personnages incroyablement colorée 
que l'on sait. Cependant, il n'est pas possible de faire l'économie de l'analyse du rôle central joué par le père dans cette œuvre, présence toujours structurellement moins visible, moins figurable, comme je l'ai souligné en commençant, puisqu'elle relève du domaine du symbolique. Pour prendre un exemple ailleurs, personne ne se souvient en général que la grande pièce des Yuan Le Pavillon de l'ouest (Xixiang ji) est placée d'un bout à l'autre à l'ombre d'un "personnage» invisible qui n'est autre que... le père de la jeune fille, mort, allongé dans son cercueil, et sans la présence duquel il n'y aurait tout simplement pas d'histoire.

Je n'ai fait ici qu'esquisser cette problématique du père comme élément central chez Pu Songling. Cette approche devrait se poursuivre autour d'au moins un problème que j'ai lui aussi seulement effleuré, en posant une question à laquelle je n'ai pas répondu: pourquoi ce rapport au corps? C'est que «le corps lui-même est, d'origine, ce lieu de l'Autre, en tant que c'est là que, d'origine, s'inscrit la marque en tant que signifiant. [...] [Le sujet] se fonde dans cette marque du corps qui le privilégie, qui fait que c'est la marque - la marque subjective - qui désormais domine tout ce dont il va s'agir pour ce corps ${ }^{80}$ ». Cette question du corps, Lacan n'a cessé d'y revenir, soulignant que le signifiant vient s'inscrire sur le corps, et même comme un «tatouage ${ }^{81}{ }^{1}$. Ceci est cohérent avec la logique du symptôme en psychanalyse, dans laquelle le corps souffre... de signifiants - lesquels proviennent nécessairement de l'Autre, puisqu'il ne saurait par définition y avoir de signifiant autoproduit. Si le traumatisme «ne cesse pas de ne pas s'écrire», les formations de l'inconscient, le rêve, «ça ne cesse pas de s'écrire ${ }^{82} \gg$. Dans la logique d'écriture, de marquage, le corps est bien du domaine de l'Autre, ce corps auquel il arrive bien des choses bizarres, dans le Liaozhai zhiyi. Une autre étape à explorer en cohérence avec la marque du père de Pu Songling serait le rapport du corps au symptôme, en poursuivant dans des voies qui ont déjà été dégagées avec compétence autour de notions dont je n'ai rien pu dire ici : celles d' «obsession», de «folie»... (notions de pi, de $\left.c h i^{83}\right)$, qui posent la question du texte par rapport à l'inscription du symptôme de son auteur.

80. Lacan (1966-1967), 31/05/1967. Voir Pluth (2008): 16.

81. Lacan (1973) : 159, 22/04/1964. Voir Pluth (2008) : 59-60.

82. Didier-Weill (2010): 49.

83. Zeitlin (1993) : 61-97, chap. «Obsession»; Weightman (2008); Chiang (2005). 


\section{BibLIOGRAPHIE}

Askofaré Sidi et SAuret Marie-Jean (2004). La question du père: père et symptôme. L'Évolution psychiatrique, $\mathrm{n}^{\circ}$ 69: 257-278.

Assoun Paul-Laurent (2009). Corps et symptôme. Paris: Anthropos, «Leçons de psychanalyse».

Aubert Jacques (dir.) (1987). Joyce avec Lacan. Paris : Navarin, «Bibliothèque des Analytica ».

BARR Allan (1984). The Textual Transmission of Liaozhai Zhiyi. Harvard Journal of Asiatic Studies, ${ }^{\circ} 44$ (2) : 515-562.

BARR Allan (1985). A comparative study of early and late tales in Liaozhai Zhiyi. Harvard Journal of Asiatic Studies, ${ }^{\circ} 45$ (1): 157-202.

BARr Allan (1986). Pu Songling and the Qing Examination System. Late Imperial China, $\mathrm{n}^{\circ} 7$ (1): 87-109.

BARR Allan (1989). Disarming intruders, alien women in Liaozhai zhiyi. Harvard Journal of Asiatic Studies, $\mathrm{n}^{\circ} 49$ (2): 501-517.

BARr Allan (2007a). Liaozhai Zhiyi and Chinese Vernacular Fiction. In Daria BerG (dir.), Reading China: Fiction, History and the Dynamics of Discourse - Essays in Honour of Professor Glen Dudbridge. Leiden/Boston: Brill, «China studies», $10: 3-36$.

BARr Allan (2007b). Liaozhai Zhiyi and Shiji. Asia Major, 3rd series, n 20 : 133-153.

Benslama Fethi (2004). La Psychanalyse à l'épreuve de l'Islam. Paris: Flammarion, «Champs».

BRIVIC Sheldon (2008). Joyce through Lacan and Žižek: Explorations. New York: Palgrave Macmillan, «New directions in Irish and Irish American literature».

Bruno Pierre (2006). L'arrangement (sur la perversion). Psychanalyse, $\mathrm{n}^{\circ} 5$ (1): 5-18.

Bruno Pierre, Bruno Catherine, Aoulllé Sophie et al. (2008). Père et Nom(s)-du-Père (1 ${ }^{\mathrm{re}}$ partie). Psychanalyse, $\mathrm{n}^{\circ} 12$ (2): 101-113.

Bulat-Manenti Gorana (2009). Duplicité paternelle et question de la filiation en psychanalyse. La Clinique lacanienne, $\mathrm{n}^{\circ} 16$ (2): 181-190.

Chang Chun-shu et Chang Shelley Hsueh-lun (1998). Redefining History: Ghosts, Spirits, and Human Society in P'u Sung-ling's World, 1640-1715. Ann Arbor: University of Michigan Press.

ChIAng Sing-chen Lydia (2005). Collecting the Self: Body and Identity in Strange Tale Collections of Late Imperial China. Leiden/Boston: Brill, «Sinica Leidensia».

Dars Jacques et Chan Hing-ho (dir.) (2001). Comment lire un roman chinois. Arles: Picquier.

DidIER-WEILl Alain (2010). Écriture de l'aleph. Figures de la psychanalyse, no 19 (1):4552.

Dor Joël (2002). Introduction à la lecture de Lacan, vol. I et II : L'inconscient structuré comme un langage. La structure du sujet. Paris: Denoël.

Elman Benjamin A. (2000). A Cultural History of Civil Examinations in Late Imperial China. Berkeley: University of California Press.

Essaim, $n^{\circ}$ 16: Des folies et des auvres (2006/1). 
FREUd Sigmund (1981). Au-delà du principe de plaisir. In Essais de psychanalyse, Jean Laplanche et Jean-Bertrand Pontalis (trad.). Paris: Payot: 52-59.

Freud Sigmund (1986a). L'Homme Moïse et la religion monothéiste, trois essais. Paris : Gallimard, «Connaissance de l'inconscient».

Freud Sigmund (1986b). Le Délire et les rêves dans la Gradiva de W. Jensen. Paris: Gallimard, «Connaissance de l'inconscient».

FreUd Sigmund (1993). Totem et Tabou. Quelques concordances entre la vie psychique des sauvages et celle des névrosés. Paris: Gallimard, «Connaissance de l'inconscient».

FREUd Sigmund (2002). Contribution à la psychologie de la vie amoureuse, II. Sur le plus général des rabaissements de la vie amoureuse. In La Vie sexuelle, Denise BERGER, Jean LAPlanche et al. (trad.). Paris: Presses universitaires de France: 55-65.

Freud Sigmund (2003). Analyse d'une phobie chez un petit garçon de 5 ans (Le petit Hans). In Cinq psychanalyses, Marie Bonaparte et Rudolph Maurice Loewenstein (trad.). Paris : Presses Universitaires de France, «Bibliothèque de psychanalyse»: 93-198.

FREUd Sigmund (2006). CEuvres complètes: psychanalyse. Volume VI, 1901-1905: Trois essais sur la vie sexuelle. Pierre Cotet, Janine Altounian, Pascale Haller et al. (trad.). Paris: Presses Universitaires de France.

Guan Wu 关兀 (1996). Liaozhai zhiyi fuchou zhuti qianlun《聊斋志异》复仇主题 浅论 (1/2). Shiyan zhiye jishu xueyuan xuebao 十堰职业技术学院学报, $n^{\circ} 27$ 31(4).

Guan $\mathrm{Wu}$ 关兀 (1997). Liaozhai zhiyi fuchou zhuti qianlun (xu) 《聊斋志异》复仇主 题浅论(续) (2/2). Shiyan zhiye jishu xueyuan xuebao 十堰职业技术学院学报, $\mathrm{n}^{\circ} 2: 40-44$.

Hanyu da cidian 漢語大詞典 (1997). Shanghai: Hanyu da cidian chubanshe 漢語大詞 典出版社 (Suoyin ben 縮印本), 3 vol.

Hanyu da zidian 漢語大字典 (1993). Chengdu : Sichuan Cishu chubanshe 四川辭書出 版社, Hubei Cishu chubanshe 湖北辭書出版社.

Ho Kin-chung (1988). Nezha, figure de l'enfant rebelle. Études chinoises, nº 7-2:7-26.

Huntington Rania (2000). Foxes and Sex in Late Imperial Chinese Narrative. Nan Nü : Men, Women, and Gender in Early and Imperial China, $\mathrm{n}^{\circ} 2(1)$ : 78-128.

Huntington Rania (2003). Alien Kind: Foxes and Late Imperial Chinese Narrative. Cambridge, Mass.: Harvard University Asia Center, Harvard University Press, «Harvard East Asian monographs».

Jiaoyubu guoyu tuixing weiyuanhui (Taiwan) 教育部國語推行委員會 (台灣) (ministère de l'Éducation [Taiwan], Comité de promotion de la langue nationale), 1994. Chongbian Guoyu cidian xiudingben 重編國語辭典修訂本 (Dictionnaire de la langue chinoise, nouvelle édition révisée). URL: http://dict.revised.moe. edu.tw/index.html.

LaCAN Jacques (1966-1967). Le Séminaire, livre XIV : La logique du fantasme. 19661967. Inédit.

LaCAN Jacques (1973). Le Séminaire, livre XI: Les quatre concepts fondamentaux de la psychanalyse. 1964. Jacques-Alain Miller (éd.). Paris: Éditions du Seuil.

LACAN Jacques (1975). Le Séminaire, livre XXII : R.S.I. 1975. Jacques-Alain MiLler (éd.). 
LaCAn Jacques (1986). Le Séminaire, livre VII : L'éthique de la psychanalyse, 19591960. Jacques-Alain Mirler (éd.). Paris: Éditions du Seuil.

LACAN Jacques (1994). Le Séminaire, livre IV : La relation d'objet. 1956-1957. JacquesAlain Miller (éd.). Paris : Éditions du Seuil.

LACAN Jacques (1998). Le Séminaire, livre V: Les formations de l'inconscient, 19571958. Jacques-Alain Miller (éd.). Paris : Éditions du Seuil.

LACAN Jacques (1999a). Écrits. Paris: Seuil, «Points Essais», 2 vol.

LACAN Jacques (1999b). Fonction et champ de la parole et du langage en psychanalyse. In Écrits. Paris: Seuil, «Points Essais » : 235-321.

LaCAn Jacques (2005). Le Séminaire, livre XXIII : Le sinthome. 1975-1976. JacquesAlain Miller (éd.). Paris: Seuil.

LÉVy André (Lei Wei’an 雷威安) (1992). Man tan Liaozhai zhiyi «Zizhi» 漫谈聊斋 志异《自志 》. In Gu Meigao 辜美高 et WANG Zhizhong 王枝忠 (dir.), Guoji Liaozhai lunwenji 国际聊斋论文集. Beijing: Beijing shifanxueyuan chubanshe: 276-281.

MA Ruifang 马瑞芳 (1986). Pu Songling ping zhuan 蒲松龄评传. Beijing: Renmin wenxue.

MA Ruifang 马瑞芳 (2001). Cong «Shanmeng zhi fu» dao «li hun zhi zi»-Liaozhai renwu tan 《从《善梦之父》到《离魂之子》-一聊斋人物谈. Wenshi zhishi 文史 知识, $\mathrm{n}^{\circ} 239$ (5): 77-82.

McMahon Keith (2006). The Remarkable Woman in Pu Songling's Liaozhai zhiyi: A Theorization of Qing. In Paolo Santangelo et Donatella Guida (dir.), Love, Hatred, and Other Passions: Questions and Themes on Emotions in Chinese Civilization. Leiden/Boston: Brill: 212-228.

McMahon Keith (Ma Kemeng 馬克夢) (2007). Qinüzi de nanban yu nüxing qizhi de bentilun 奇女子的男伴與女性氣質的本體論. Tsinghua Journal of Chinese Literature, Qinghua zhongwen xuebao 清華中文學報, $\mathrm{n}^{\circ}$ 1:315-326.

McMahon Keith (2009). Polygamy and Sublime Passion: Sexuality in China on the Verge of Modernity. Honolulu: University of Hawai'i Press.

Pluth Ed (2008). Signifiers and Acts: Freedom in Lacan's Theory of the Subject. New York: State University of New York Press, «SUNY Series, Insinuations, Philosophy, Psychoanalysis, Literature».

Pommier Gérard (2003). Respiration du symptôme. La Clinique lacanienne, $\mathrm{n}^{\circ} 6$ (1): 97-120.

Pommier Gérard (2005). Du monstre phobique au Totem, et du Totem au Nom-du-Père. La Clinique lacanienne, $\mathrm{n}^{\circ} 9$ (2):21-46.

Porge Erik (1997). Les Noms du père chez Jacques Lacan - Ponctuations et problématiques. Toulouse: Érès.

PoRret Philippe (2010). Compte rendu sur Rainier Lanselle, Trois contes étranges (2009). Les Lettres de la Société de psychanalyse freudienne, $\mathrm{n}^{\circ}$ 23. Paris: Campagne première: 251-253.

Pu Songling et Lanselle Rainier (trad.) (2009). Trois contes étranges, 2 vol. Paris: Presses Universitaires de France, «Sources, Fondation Martin Bodmer - Genève». 
Pu Songling et Lévy André (trad.) (2005). Chroniques de l'étrange, 2 vol. Arles: Picquier.

Pu Songling et Minford John (trad.) (2006a). Strange Tales from a Chinese Studio. Londres/New York/Toronto: Penguin Classics.

Pu Songling et Minford John (trad.) (2006b). Strange Tales from a Chinese Studio. Londres/New York/Toronto: Penguin Classics, livre électronique, 8151 écrans.

Pu Songling de 《yuefu 《蒲松龄的《越幅》(anon.). Relevé le 29 avr. 2011, www. yidudu.com/read_2254_258656.html

Pu Songling et GILEs Herbert (trad.) (1916). Strange Stories from a Chinese Studio. Kelly \& Walsh.

Pu Songling 蒲松齡 et ZHANG Youhe 張友鶴 (éd.) (1978). Liaozhai zhiyi, Huijiao huizhu huiping ben 聊齊志異, 會校會注會評本, 4 vol., Shanghai: Shanghai guji chubanshe.

RivierE Joan (1929). Womanliness as Masquerade. International Journal of Psychoanalysis, $\mathrm{n}^{\circ}$ 10: 303-313.

SAFOUAN Moustapha (dir.) (2001). Lacaniana: les séminaires de Jacques Lacan (I, 1953-1963). Paris : Fayard.

VAnIER Alain (2000). Lacan. Paris: Les Belles Lettres, «Figures du savoir», 11.

WANG Fuchen 王福臣 (2010). Chikuang yu fanpan de liangji rensheng: Qianxi Pu Songling keju xintai bianqian 痴狂与反叛的两极人生一一浅析蒲松龄科举心 态变迁. Pu Songling yanjiu 蒲松龄研究, $\mathrm{n}^{\circ}$ 4: 5-13.

WANG Zhizhong 王枝忠 (1990). Pu Songling lunji 蒲松龄论集. Beijing: Wenhua yishu chubanshe.

Weightman Frances (2006). Folly (chi 癡) in the Liaozhi Zhiyi 仰齋誌異. In Paolo SANTANGElo et Donatella Guida (dir.). Love, Hatred, and Other Passions : Questions and Themes on Emotions in Chinese Civilization, Leiden/Boston: Brill: 197-211.

Weightman Frances (2008). The Quest for the Childlike in Seventeenth-century Chinese Fiction: Fantasy, Naivety, and Folly. Lewiston, NY: Edwin Mellen Press.

Wu Pei-yi (1990). The Confucian's Progress : Autobiographical Writings in Traditional China. Princeton: Princeton University Press.

YUAn Shishuo 袁世硕 et Xu Zhongwei 徐仲伟 (2000). Pu Songling pingzhuan 蒲松 龄评传. Nanjing: Nanjing daxue chubanshe, «Zhongguo sixiangjia pingzhuan congshu»中国思想家评传从书.

Zafiropoulos Markos (2003). Lacan et Lévi-Strauss ou le retour à Freud (1951-1957). Paris : Presses Universitaires de France.

ZeItlin Judith T. (1993). Historian of the Strange: Pu Songling and the Chinese Classical Tale. Stanford: Stanford University Press.

Zeitlin Judith T. (2007). The Phantom Heroine: Ghosts and Gender in Seventeenthcentury Chinese Literature. Honolulu: University of Hawai'i Press. 
Rainier Lanselle

\section{GlossaIRE}

Bi 畢

chi 璇

Deng Chengde 鄧成德

《Dong-sheng» 董生

《Egui» 餓鬼

Erlang shen二朗神

«Fang Wenshu» 房文淑

Fengshen yanyi 封神演義

《Hua pi»畫皮

《Jiangcheng» 江城

jin bang ti ming 金榜題名

jinshi 進士

《Jinü»績女

$k e j u$ 科舉

Liaozhai zhiyi 聊齋誌異

《Liaozhai zizhi» 聊齋自誌

Lou 婁 (dame $\sim)$

Mulian 目連

Nezha 哪吒

$p i$ 癖

pianwen 駢文

Pu Songling 蒲松齡 (1640-1715)

《Quan xue pian» 勸學篇

Ruyu 如玉

shengyuan 生員

《Shuchi 》 書癡

Shuzhong zi you huangjin wu 書中自有黄金屋 [...] Shuzhong zi you yan ru yu 書中 自有顏如玉

Song Zhenzong (Empereur) 宋真宗 (r. 997-1022)

《Xi Fangping》席方平

xiangshi 鄉試

Xixiang ji 西廂記

Yan 顏

Yan Ruyu 顏如玉

Yansheng 充生

Yanzhou 充州

«Yu Qu’e»于去惡 